\title{
Easing the pain: challenges and opportunities in headache management
}

Headache is common. The lifetime prevalence of headache in the UK general population is over $90 \%{ }^{1}$ and an estimated $46 \%$ of the population are troubled by headache in any one year. ${ }^{2}$

Headache accounts for $4.4 \%$ of consultations in primary care ${ }^{3}$ and $20-30 \%$ of neurology out-patient consultations. ${ }^{4,5}$ Nearly all headache is primary, with the majority of patients suffering from tension-type headache, migraine, or both together. Secondary headaches, that is, headaches attributed to another disorder, are rare. There is no diagnostic test for primary headache. Diagnosis is made by taking a good history. The vast majority of patients with headache are managed by GPs, and only $2-3 \%$ are referred to neurology services. ${ }^{3}$

Despite this, GPs seem to be poor at diagnosing headache. In this issue of the $B J G P$, Kernick et $a^{6} /$ report on a large cohort of patients presenting with new onset headache in primary care in the UK. They found that $70 \%$ were not given a diagnosis; $24 \%$ were diagnosed with either migraine, tension headache, or cluster headache; and $6 \%$ were diagnosed with secondary headache. This is similar to previous research. Primary care research, such as the Landmark $^{7}$ and Spectrum studies, ${ }^{8}$ has shown that migraine is underdiagnosed and that tension-type headache and sinus headache are overdiagnosed.

The picture is similar in secondary care, with acute headache being wrongly diagnosed in receiving medical units and A\&E departments (DP Breen, unpublished data, 2008). In an editorial in the July 2006 issue of the Journal, ${ }^{9}$ Kernick described how patients with cluster headache often see many different specialists, ranging from ENT surgeons, ophthalmologists, and general physicians before they are diagnosed as having cluster headache.

Even if the correct diagnosis is made, many patients are not managed optimally despite the availability of effective therapies. GPs often use medications that they are familiar with, rather than choosing the most appropriate therapy based on headache type, severity, and associated symptoms. Even if appropriate acute therapy is chosen, patients are often not advised about the correct use.

With respect to migraine prophylaxis, a large American study ${ }^{10}$ showed only $12 \%$ of migraine patients are prescribed prophylaxis, yet 50\% would be eligible for it. In this issue of the Journal, Kol et a/11 report that in a cohort of Dutch migraine patients eligible for prophylaxis, only $8 \%$ were prescribed it, yet $55 \%$ would use it if offered the option. They conclude that physicians can play a more active role in optimising migraine therapy for their patients.

Many clinicians are not aware that using opiate-based or triptan medications for more than 10 days' a month, or simple analgesics such as aspirin and ibuprofen for more than 15 days' a month, can cause headache. Half of all patients with chronic headache, defined as headache on more than 15 days' a month, overuse medication. Opiates or opiate combinations have no place in routine headache management.

Effective diagnosis and management of primary headache is important, not only because headache causes anxiety for patients and doctors but also because primary headache causes significant morbidity. Migraine is the most common severe primary headache, affecting 6 million people in the UK, and it causes significant disability with over 100000 people absent from work or school every working day. ${ }^{12}$ The World Health Organization ranks migraine in its top 20 disabling conditions for females aged 15-44 years. ${ }^{13}$ Although tension-type headache is generally less burdensome than migraine, the total societal burden of tensiontype headache is higher than migraine due to its much higher prevalence. Chronic headache affects $4 \%$ of the UK population ${ }^{14}$ and also causes significant disability.

It is unclear why GPs find headache diagnosis and management difficult. It is likely not to be just a problem of cognitive knowledge but of how this knowledge is applied in the consulting room. The Global Campaign to Reduce the Burden of Headache Worldwide, known as 'Lifting the Burden' (www.l-t-b.org), is a joint action between the World Health Organization, the World Headache Alliance, the International
Headache Society, and the European Headache Federation. The Lifting the Burden campaign statement is: "that the basis of the healthcare solution for headache in most parts of the world is education'. This education should first create awareness that headache disorders are a medical problem requiring treatment. Secondly, education of healthcare providers should encompass correct recognition, diagnosis, and treatment of common headache disorders.

Kernick suggests that GPs may require a number of consultations to establish a definitive headache diagnosis. However, many patients are not followed up. This may be because headache symptoms are often perceived by GPs as being difficult to diagnose and, therefore, time intensive, especially when it is presented as part of a list or as a 'by-the-way' statement at the end of a consultation.

Some GPs take the view that no one ever dies from migraine or tension headache so the patient is given a painkiller and not reviewed. Even when a review appointment is offered, research shows that many patients don't come back as they feel the doctor has not listened to them or has nothing else to offer them. ${ }^{15}$

Some patients are referred for brain scanning due to GP and patient anxiety about brain tumours. ${ }^{16}$ Patients with normal scans may be reassured but are often not given a definitive diagnosis to their original problem. Inappropriate computerised tomography not only results in unnecessary radiation exposure but can also identify incidental findings of doubtful clinical significance, such as small benign tumours, aneurysms, and arteriovenous malformations, the so called 'VOMITs' or victims of modern imaging techniques. This can cause patient concern, dilemmas about further management, potentially unnecessary procedures with associated morbidity, and problems obtaining life insurance policies.

Ridsdale et $a l^{17}$ showed that patients referred to secondary care have the same headache impact and disability as those managed in primary care. Thus, in principle, the majority of patients with episodic migraine and tension headache can be 
diagnosed and managed by GPs at practice level. Patients with chronic migraine, medication-overuse headache, and cluster headache are more difficult to manage and some will need specialist input. However, they can still be managed in primary care at a local level by GPs with a special interest in headache.

Patient feedback for these services is good and many patients comment that they value seeing a doctor with a headache interest rather than attend a general neurology clinic and consult a neurologist who has no headache interest. They feel that someone is listening to them, taking them seriously and not dismissing them as having a trivial problem. Doctors with an interest in headache often have headache themselves or partners with headache. Perhaps familiarity with headache disability and how it impacts on sufferers and their family's lives helps to create empathy in the consultation. Managing headache is not just simply about making a diagnosis and prescribing the correct drugs. Exploration of psychosocial issues that have an impact on causality and disability are equally important. Patients are more likely to allow doctors to explore the psychosocial issues if empathy has been established.

A UK-wide survey in 1999 showed that GP knowledge of headache types and awareness of guidelines was poor. ${ }^{18}$ The survey suggested that postgraduate training in headache was inadequate. No recent survey has been conducted, but it is unlikely the position has changed much. On a positive note, in the last 2 years GPs with special interest in headache services have started to raise awareness among local GPs. Although there are only about 15 of these services in the UK, approximately half of them organise local postgraduate educational meetings at regional and practice level. A Turkish study has shown that educational programmes for GPs on migraine and tension headache can increase diagnostic accuracy from $56 \%$ to $81 \% .{ }^{19}$ These headache services have agreed referral criteria with local practices and have developed resources that include doctor and patient educational leaflets, telephone, written, and email advice services, and advice on how to use headache tools such as headache diaries, impact questionnaires and structured questionnaires. Before these initiatives, many GPs were not aware of these validated tools that aid diagnosis and make best use of precious consulting time.

At a national level, the British Association for the Study of Headache (BASH; www.bash.org.uk) organises study days, and the Migraine in Primary Care Advisors group (MIPCA; www.mipca.org.uk) is developing a university diploma course. BASH and MIPCA have well-written, comprehensive headache management guidelines on their respective websites. However, both organisations are not widely known by doctors other than those interested in the area of headache.

Later this year, the Scottish Intercollegiate Guidelines Network (SIGN; www.sign.ac.uk) will launch a comprehensive evidence-based peer reviewed primary headache guideline. There will be sections on diagnosis, management, and guidance on the use of brain scanning. The aim of the SIGN guideline is to raise headache awareness and to give clinicians guidance on how to help patients manage their headaches more effectively. There is currently no agreed national standard for headache management to benchmark care against. The NHS in Scotland will be starting work on national standards later this year, and it is hoped once these standards are published headache care for patients will improve.

In summary, although some patients' headaches are diagnostically challenging and their management difficult, these patients are a minority. For most patients the diagnosis can be made by taking a careful history, effective therapies are available, and appropriate management can reduce headache disability significantly. These patients are often very grateful for receiving treatment, making work in this area a rewarding experience for the doctor.

\section{David PB Watson}

GP, Hamilton Medical Group, and GP with special interest in headache, Department of Neurology, Aberdeen Royal Infirmary, Foresterhill, Aberdeen

\section{Competing interests}

David Watson is a member of both BASH and MIPCA. He currently chairs the SIGN headache guideline group and is a member of the Scottish Neurosciences Group considering headache standards. He has been paid to lecture about headache by NHS Education For Scotland, RCGP, GSK, MSD, Menarini, Pfizer, and AstraZeneca.

\section{REFERENCES}

1. Boardman H, Thomas E, Croft PR, Millson DS Epidemiology of headache in an English district. Cephalalgia 2003; 23(2): 129-137.
2. Stovner LJ, Hagen K, Jensen R, et al. The global burden of headache: a documentation of headache prevalence and disability worldwide. Cephalalgia 2007; 27(3): 193-210.

3. Latinovic R, Gulliford M, Ridsdale L. Headache and migraine in primary care: consultation, prescription, and referral rates in a large population. J Neurol Neurosurg Psychiatry 2006; 77(3): 385-387.

4. Larner AJ. Guidelines for primary care headache disorders in primary care: an 'intervention' study. Headache Care 2006; 3(1): 1-2.

5. Patterson V, Esmonde TF. Comparison of the handling of neurological outpatient referrals by general physicians and neurologist. J Neurol Neurosurg Psychiatry 1993; 56(7): 830.

6. Kernick D, Stapley S, Hamilton W. GPs' classification of headache: is primary headache underdiagnosed? $\mathrm{Br} J \mathrm{Gen}$ Pract 2008; 58(547): 102-104.

7. Tepper SJ, Dahlof CG, Dowson A, et al. Prevalence and diagnosis of migraine in patients consulting their physician with a complaint of headache: data from the Landmark Study. Headache 2004; 44(9): 856-864.

8. Lipton RB, Cady RK, Stewart WF, et al. Diagnostic lessons from the spectrum study. Neurology 2002; 58(9 Suppl 6): S27-S31.

9. Kernick D. Matharu MS. Goadsby PJ. Cluster headache in primary care: unmissable, underdiagnosed and undertreated. Br J Gen Pract 56(528): 486-487.

10. Diamond S, Bigal M E, Silberstein S, et al. Patterns of diagnosis and acute and preventive treatment for migraine in the United States: results from the American Migraine Prevalence and Prevention Study. Headache 2007; 47(3): 355-363.

11. Kol CMC, Dekker F, Knuistingh Neven A, et al. Acceptance or rejection of prophylactic medicine in patients with migraine: a cross-sectional study. Br J Gen Pract 2008; 58(547): 98-101.

12. Steiner T, Scher AI, Stewart WF, et al. The prevalence and disability burden of adult migraine in England and their relationships to age, gender and ethnicity. Cephalalgia 2003 23(7): 519-527.

13. World Health Organization. The World Health Report 2001 Mental health: new understanding, new hope. Geneva: World Health Organization, 2001.

14. British Association for the Study of Headache. Guidelines for all healthcare professionals in the diagnosis and management of migraine, tension-type, cluster and medication-overuse headache. 3rd edn. Hull: British Association for the Study of Headache, 2007.

15. Lipscombe SL. Why do migraine patients lapse from followup? Headache Care 2004; 1(3): 191-193.

16. Morgan M, Jenkins L, Ridsdale L. Patient pressure for referral for headache: a qualitative study of GPs' referral behaviour. BJGP 2007; 57(534): 29-35.

17. Risdale L, Clark LV, Dowson AJ, et al. How do patients referred to neurologists for headache differ from those managed in primary care? BJGP 2007; 57(538): 388-395.

18. Watson D, Williams D. Investigating the need for general practitioner headache education [abstract]. 4th Congress of the European Federation of Neurological Societies. Lisbon, Portugal, September 7-11, 1999. Eur J Neurol 1999; 6 Suppl 3: 1-197.

19. Karli N, Zarifoglu M, Erer S, et al. The impact of education on the diagnostic accuracy of tension-type headache and migraine: a prospective study. Cephalalgia 2007; 27(1): 41-45.

DOI: 10.3399/bjgp08X264009

\section{ADDRESS FOR CORRESPONDENCE}

David PB Watson

Hamilton Medical Group, 4 Queens Road,

Aberdeen, AB15 4ZT

E-mail: david.watson@nhs.net 